$$
\text { BIOINFORMATION }
$$$$
\text { Discovery at the interface of physical and biological sciences }
$$ \\ BIOMEDICAL

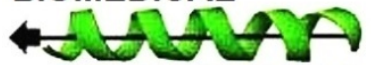 \\ INFORMATICS \\ WWW.bioinformation.net \\ Volume 18(1)
}

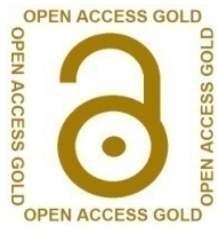

Research Article

Received November 22, 2021; Revised December 10, 2021; Accepted December 16, 2021, Published January 31, 2022

Declaration on Publication Ethics:

DOI: $10.6026 / 97320630018053$

The author's state that they adhere with COPE guidelines on publishing ethics as described elsewhere at https://publicationethics.org/. The authors also undertake that they are not associated with any other third party (governmental or non-governmental agencies) linking with any form of unethical issues connecting to this publication. The authors also declare that they are not withholding any information that is misleading to the publisher in regard to this article.

Declaration on official E-mail:

The corresponding author declares that official e-mail from their institution is not available for all authors

License statement:

This is an Open Access article which permits unrestricted use, distribution, and reproduction in any medium, provided the original work is properly credited. This is distributed under the terms of the Creative Commons Attribution License

Comments from readers:

Articles published in BIOINFORMATION are open for relevant post publication comments and criticisms, which will be published immediately linking to the original article without open access charges. Comments should be concise, coherent and critical in less than 1000 words.

Edited by $P$ Kangueane

Citation: Katsuda et al. Bioinformation 18(1): 53-60 (2022)

\section{Sub-GOFA: A tool for Sub-Gene Ontology function analysis in clonal mosaicism using semantic (logical) similarity}

\section{Tadaaki Katsuda1 $^{*}$, Noriko Sato1, Kaoru Mogushi2,3, Takeshi Hase 2,3,4,5 \& Masaaki Muramatsu1 \\ ${ }^{1}$ Department of Molecular Epidemiology, Medical Research Institute, Tokyo Medical and Dental University, 24F, M\&D Tower, 1-5-45 Yushima, Bunkyo-ku, Tokyo, 113-8510, Japan; ${ }^{2}$ Institute of Education, Tokyo Medical and Dental University, 20F, M\&D Tower, 1-5-45 Yushima, Bunkyo-ku, Tokyo, 113-8510, Japan; ${ }^{3}$ Faculty of Pharmacy, Keio University, 1-5-30 Shibakoen, Minato-ku, Tokyo, 105-8512, Japan; 4The Systems Biology Institute, Saiseilkedayama Bldg. 5-10-25 Higashi Gotanda Shinagawa, Tokyo, 141-0022, Japan; 5 SBX BioSciences, Inc, 1600 - 925 West Georgia Street, Vancouver, BC V6C 3L2, Canada; Tadaaki Katsuda - Email: tadakatsuda.epi@mri.tmd.ac.jp; Phone: +81-3- 5803-4763; *Corresponding author}

\section{Author contacts:}

Tadaaki Katsuda - tadakatsuda.epi@mri.tmd.ac.jp; Noriko Sato - nsato.epi@mri.tmd.ac.jp; Kaoru Mogushi - mogushi.mds@tmd.ac.jp; Takeshi Hase - 2121adm@tmd.ac.jp; Masaaki Muramatsu - muramatsu.epi@mri.tmd.ac.jp 


\begin{abstract}
:
Clonal mosaicism (a detectable post-zygotic mutational event in cellular subpopulations) is common in cancer patients. Detected segments of clonal mosaicism are usually bundled into large-locus regions for statistical analysis. However, low-frequency genes are overlooked and are not sufficient to elucidate qualitative differences between cancer patients and non-patients. Therefore, it is of interest to develop and describe a tool named Sub-GOFA for Sub-Gene Ontology function analysis in clonal mosaicism using semantic similarity. Sub-GOFA measures the semantic (logical) similarity among patients using the sub-GO network structures of various sizes segmented from the gene ontology (GO) for clustering analysis. The sub-GO's root-terms with significant differences are extracted as disease-associated genetic functions. Sub-GOFA selected a high ratio of cancer-associated genes under validation with acceptable threshold.
\end{abstract}

Keywords: Sub-GOFA, tool, sub-gene ontology, function, clonal mosaicism, semantic, logical, similarity

\section{Background:}

Clonal mosaicism is a post-zygotic large-scale mutational event in chromosomes and mitochondria in cellular subpopulation. It occurs in the peripheral blood with aging and in tissue, which are known to be associated with cancer [1-9] and diabetes [10, 11]. Genetic function analysis of rare clonal mosaics is generally performed by bundling them into large-locus regions for statistical analysis. Thousands of genes are affected under abnormal regions in clonal mosaicism, but a large number of genes in the regions are lowfrequency genes that are uncommon among patients. Conventional statistical approaches overlook the effects of low-frequency genes and their genetic functions and are not sufficient to elucidate qualitative differences between cancer patients and non-patients. Recent advancements in high-throughput biological technologies have led to a significant accumulation of structured biological knowledge, and new approaches based on semantic technology are being attempted to exploit this information. The gene ontology (GO)[12] is one of the international projects that aims to create a common vocabulary in the field of life science research regarding the description of genetic functions. Each GO term is associated with the form of a hierarchical structure, which represents semantic relationships of inclusion. Using biological knowledge embedded in the GO structure has enabled further comparison or classification of given set of genes obtained by various omics analysis techniques (e.g., genomics, transcriptomics, and proteomics) to understand the biological phenomena? Currently, number of semantic-based tools has played an important role in improving analysis of proteomics and transcriptomics at the level of functional genomics using different semantic similarity measures among GO terms [13]. This approach has not yet been attempted for large-scale genomic regional dataset that consists of gene list in specific genomic region. The pair wise approach measures the individual semantic similarity for every pair of terms and integrates these into a global similarity measure. This global similarity measure is dependent on the size and structure of the network. Another important property of GO is a huge network structure of hierarchical directed acyclic graphs (DAGs). In clonal mosaicism, pair-wise semantic similarity measure between large-scale genomic regional datasets handles thousands of genes as variables. Even if the similarity of a characteristic genetic functions is found in a segmented specific GO network region, that similarity is homogenized within a global similarity measure in the entire GO network, and those genetic functions are overlooked.
We attempt to obtain pairwise similarity between large-scale genomic regional datasets on patients using sub-graph structures of GO networks in various sizes to implement a novel method for genetic functional analysis considering low-frequency genes and GO terms. Since GO is a hierarchical network of DAG structures, it can be segmented from higher GO terms to lower GO terms. Sub$\mathrm{GO}$ is a hierarchical network of partial genetic functions segmented from GO. By applying GO terms under each sub-GO network, we prevent homogenization of characteristic pairwise similarities between large-scale genomic regional datasets on patients in a segmented specific GO network region. By statistically evaluating the pairwise similarity between those patients, we can measure the influence of the genetic function of the sub-GO on the subject disease. In addition, sub-GO networks include all the genes, especially low-frequency genes, in GO annotations associated with patients and thus may be useful for genetic functional analysis for low-frequency genes. Therefore, it is of interest to develop and describe a tool named Sub-GOFA for Sub-Gene Ontology function analysis in clonal mosaicism using semantic similarity.

\section{Methodology: \\ Sub-GOFA algorithm:}

Figure 1A shows an overview of the Sub-GOFA algorithm. First, Sub-GOFA segments the overall GO (version 1.2) network into 43,364 Sub-GO networks. Next, Sub-GOFA measures the information criterion (IC)-based semantic similarity between largescale genomic regional datasets annotated with multiple GO terms for all patient pairs, including cancer patients and non-patients. Sub-GOFA adopted Lin's method [14] in ontology Similarity (version 2.5) [15], which were implemented in R statistical language version 3.4.3 (http://www.r-project.org/), for IC-based semantic similarity. Then, cluster analysis by the Ward's method is performed to classify all samples in a dataset into two groups based on the similarity results of all pairs of samples. Between those two groups, there may be a significant difference in the proportion of cancer patients and non-patients. In the case of those with significant differences, the Sub-GO networks could be regarded as genetic functions associated with the differences between cancer patients and non-patients. Lastly, the proportions of cancer-patients and non-patients in each cluster are statistically evaluated using the Fisher's exact test adjusted by the false discovery rate (FDR), and root terms of sub-GO with significant differences are extracted as disease-associated genetic functions.Sub-GOFA is not published, and thesource codes are available from the authors upon request. 


\section{Clonal mosaicism dataset:}

The clonal mosaicism dataset for Sub-GOFA was created using public domain datasets [1-6]. Each sample in this dataset describes the clinical phenotype information of the specific cancer name, nondisease, or other-disease name and the position information of the abnormal region of the clonal mosaicism, such as chromosome number, start position, and end position. The clinical phenotype information in our study contains the following types of solid cancer: bladder cancer $(n=61)$, prostate cancer $(n=71)$, and lung cancer $(n=163)$. Figure S1 shows flowchart of creating analysis datasets for three cancer types. Each control group was created by randomly sampling of 100 samples from the group of non-disease samples $(n=723)$ with clonal mosaicism, regardless of disease type, after adjusting for gender and age. For the lung cancer analysis dataset, 100 samples were randomly sampled from lung cancer patients. The maximum sample size of the analysis dataset was set to 200 because the calculation of similarity for all patient pairs requires massive analyses of 43,364 terms of all sub-GO, which is very computationally intensive. We annotate each sample with GO terms using biomaRt (version 3.13) [16] in the R environment from the position information, start and end in abnormal region. In total, case and control groups were combined to create each analysis datasets for the three cancer types in Sub-GOFA.

\section{Comparison with conventional statistical methods:}

Two conventional statistical methods were used to compare the analysis performance of GO terms. They are (1) Fisher's exact test for gene symbols and (2) Fisher's exact test for GO terms. The FDR thresholds of each method, including Sub-GOFA, were set to the same value, and the detection performance was evaluated by comparing the detection ratio of cancer-associated genes while changing the FDR threshold values. The cancer-associated genes were extracted from DisGeNET [17], which is known as a platform containing the largest collection of genes and variants involved in human diseases.
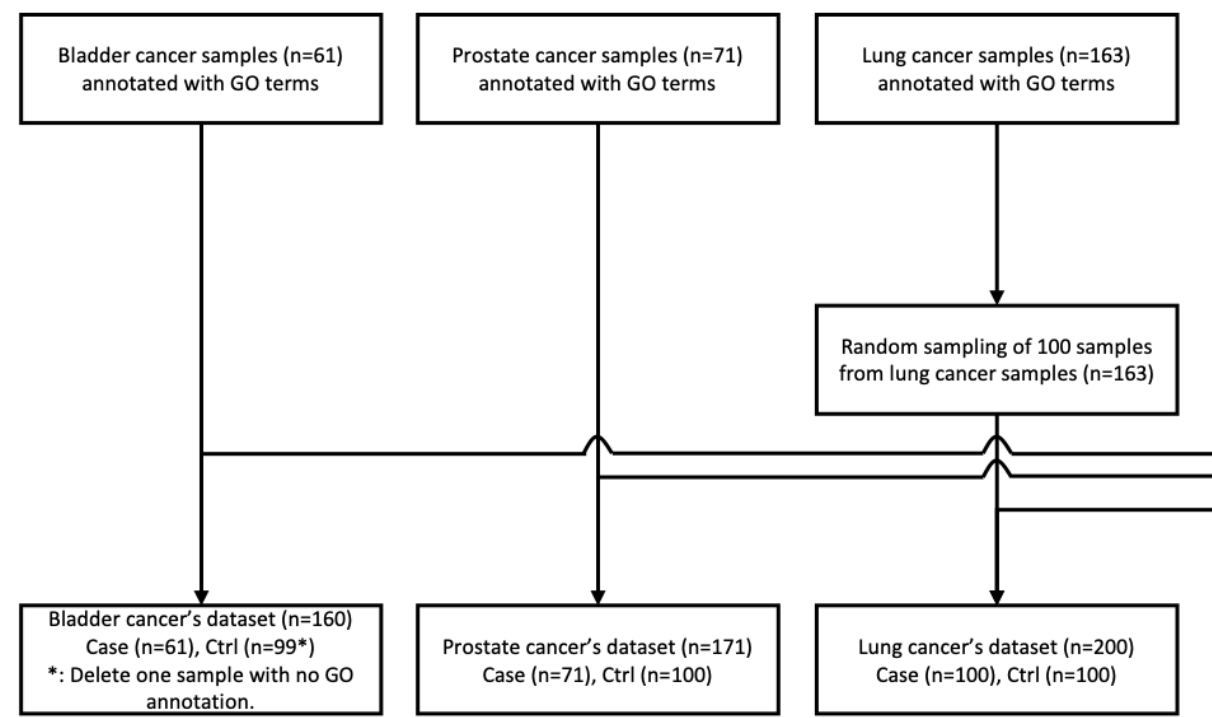

Non-disease samples $(n=723)$ with

clonal mosaicism annotated with GO terms
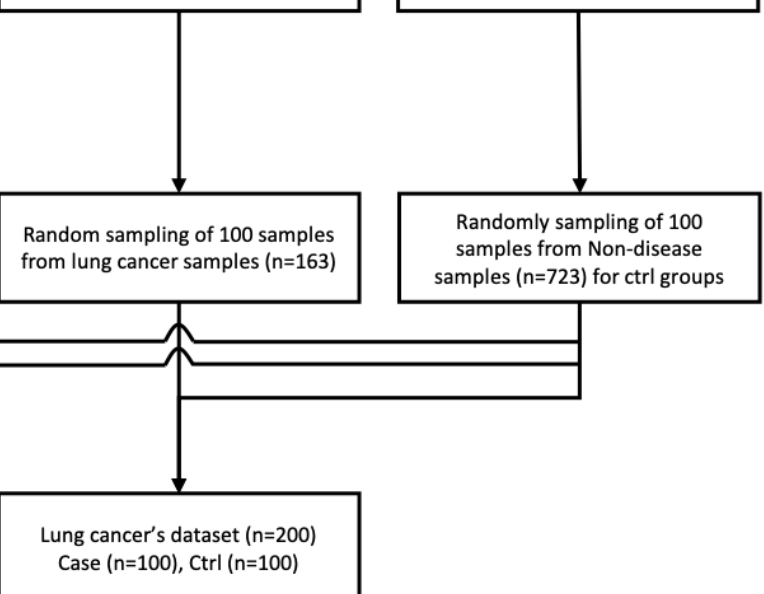
samples $(n=723)$ for $\mathrm{ctrl}$ group

Figure S1: Flowchart of creating analysis datasets for three cancer types

Table 1: Top 10 genetic functions with differences between lung cancer patients and non-patients

\begin{tabular}{llll}
\hline Ranking & Sub-GO root ID & Sub-GO root term & FDR value \\
\hline 1 & GO:0035372 & Protein localization to microtubule & 0.0373 \\
2 & GO:0031061 & Negative regulation of histone methylation & 0.0398 \\
3 & GO:0010288 & Response to lead ion & 0.0517 \\
4 & GO:0031116 & Positive regulation of microtubule polymerization & 0.1052 \\
5 & GO:0072678 & T cell migration & 0.1095 \\
6 & GO:0035564 & Regulation of kidney size & 0.1095 \\
7 & GO:0060761 & Negative regulation of response to cytokine stimulus & 0.1095 \\
8 & GO:0045600 & Positive regulation of fat cell differentiation & 0.1095 \\
9 & GO:2001244 & Positive regulation of intrinsic apoptotic signaling pathway & 0.1095 \\
10 & GO:0051570 & Regulation of histone H3-K9 methylation & 0.1095 \\
\hline
\end{tabular}


Tables S1: Basic statistics of the analysis dataset in 3 types of cancer.

\begin{tabular}{llllll}
\hline Analysis Dataset Name & & All sample & Female & Male & \multicolumn{1}{l}{ Age } \\
\hline \multirow{2}{*}{ Bladder cancer's dataset } & Case (bladder) & 61 & $13(21.3 \%)$ & $48(78.7 \%)$ & $69.01 \pm 6.71$ \\
& Ctrl (no-cancer) & 99 & $21(21.2 \%)$ & $78(79.6 \%)$ & $69.93 \pm 10.57$ \\
\multirow{2}{*}{ Prostate cancer's dataset } & Case (prostate) & 71 & 0 & $71(100.0 \%)$ & $71.01 \pm 6.72$ \\
& Ctrl (no-cancer) & 100 & 0 & $100(100.0 \%)$ & $70.16 \pm 10.82$ \\
\multirow{2}{*}{ Lung cancer's dataset } & Case (lung) & 100 & $52(52.0 \%)$ & $48(49.0 \%)$ & $67.15 \pm 9.60$ \\
& Ctrl (no-cancer) & 100 & $39(39.0 \%)$ & $61(61.0 \%)$ & $68.93 \pm 10.48$ \\
\hline
\end{tabular}

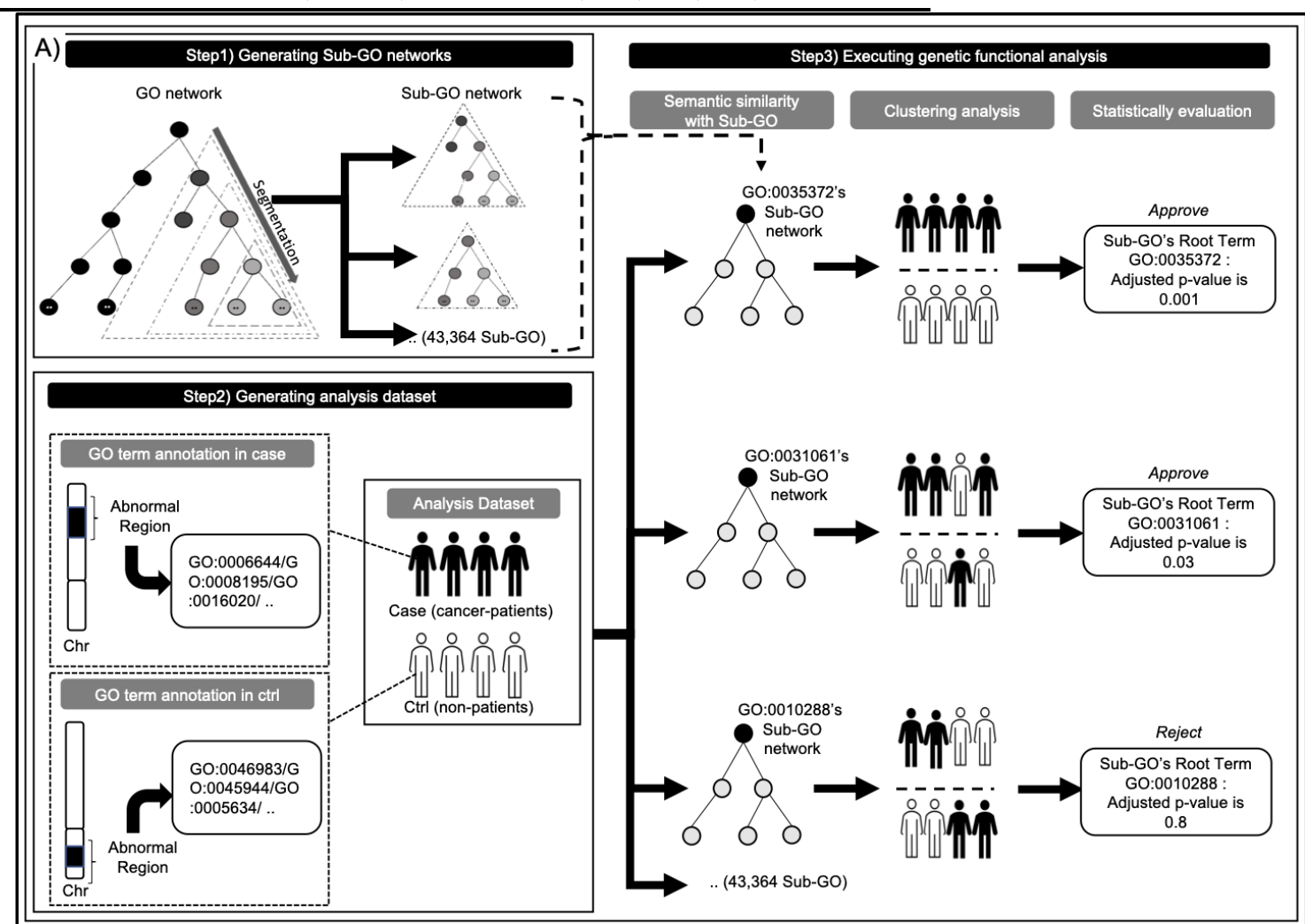

B)

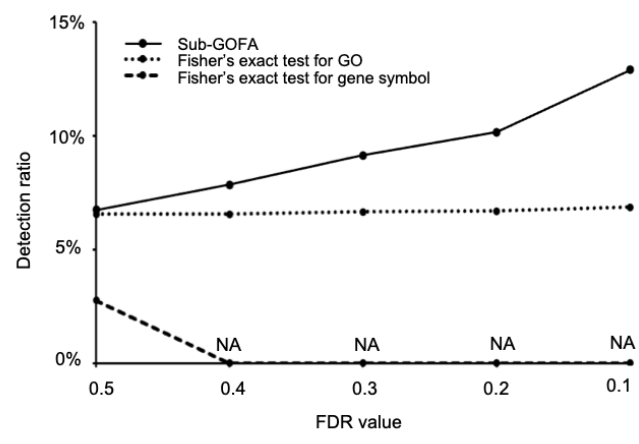

C)

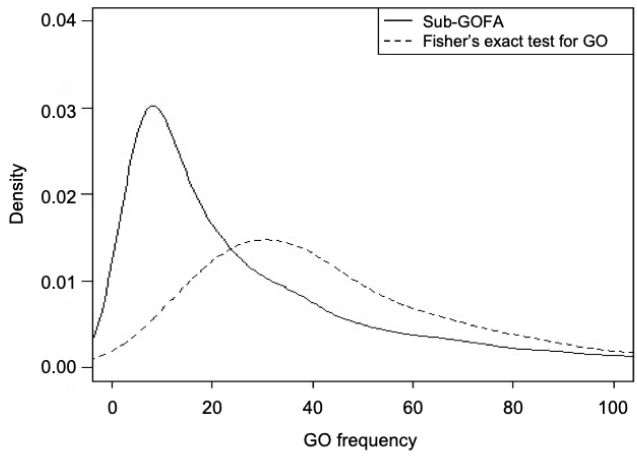

Figure 1: Sub-GOFA's genetic functional analysis overview and performance for clonal mosaicism. A: The analysis flow of Sub-GOFA. Step 1 - Generating Sub-GO networks $(43,364)$ by segmenting the huge GO network. Step 2 - Generating analysis dataset annotating multivariate GO terms in abnormal regions for case (cancer-patients) and ctrl (non-patients). Step 3 - Executing genetic functional analysis consists of semantic similarity analysis with Sub-GO $(43,364)$, clustering analysis from the similarity results and statistical evaluation with adjusted pvalues. B: Comparison of lung cancer associated gene contents ratio of Sub-GOFA and Fisher's exact test for gene symbols and for GO terms with varying FDR values. C: Plot of the frequency and density of GO terms calculated by Sub-GOFA and Fisher's exact test for GO in lung cancer analysis dataset at FDR value of 0.3 . 


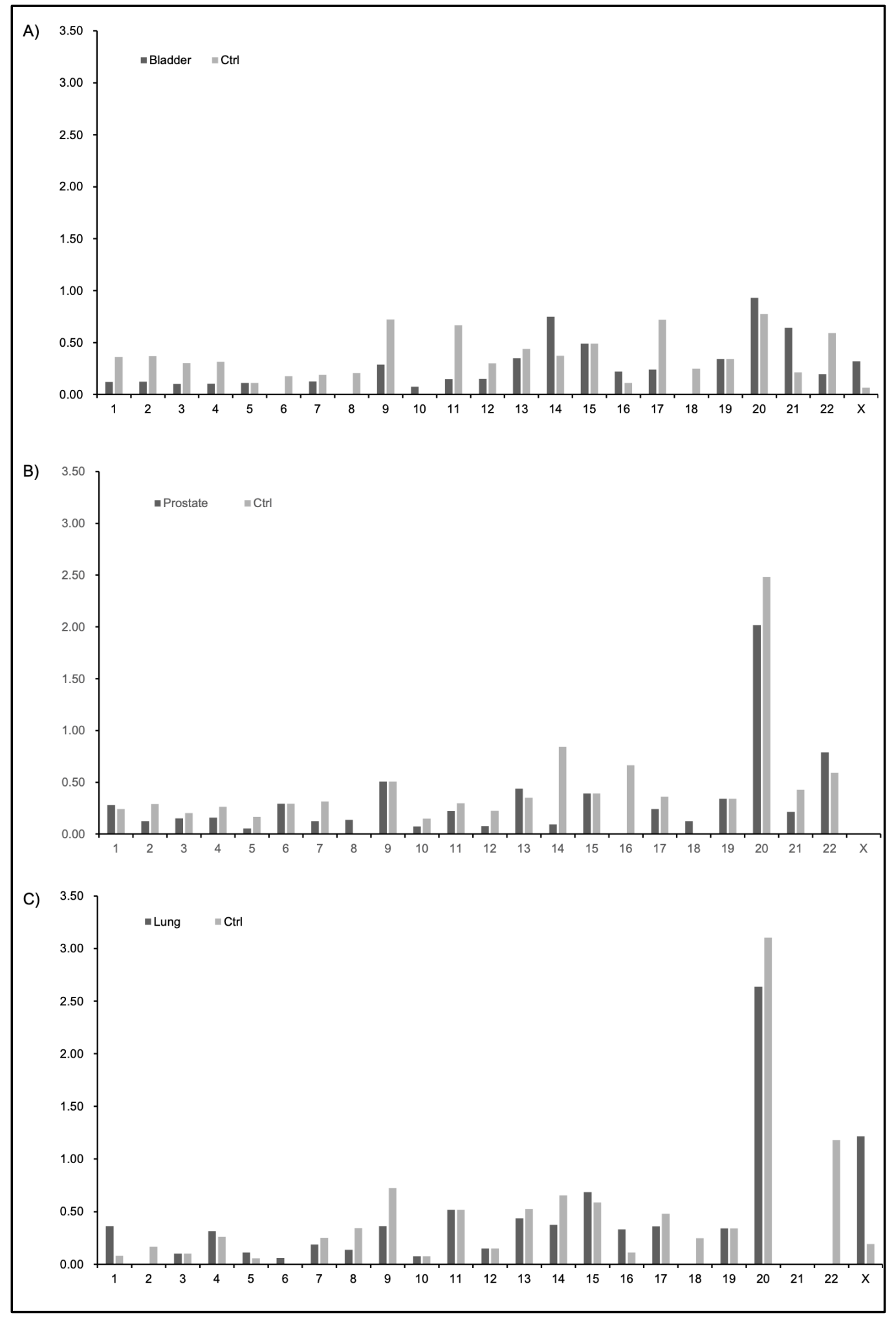

Figure 2: Comparison of case (cancer-patients) and ctrl (non-patients) for the frequency of clonal mosaicism at $1000 \mathrm{Mb}$ in each chromosome. 

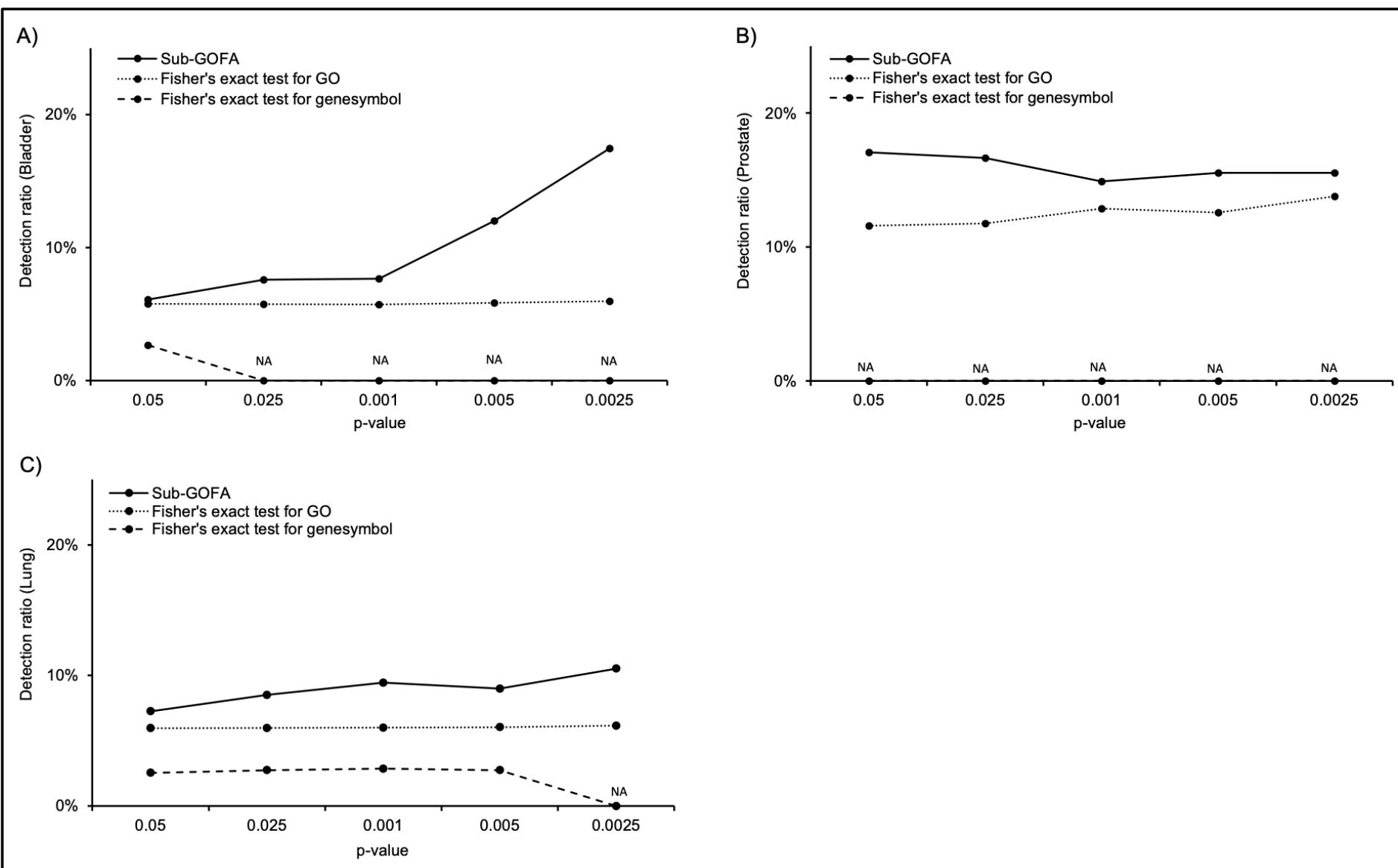

Figure 3: Comparison of detection ratio of cancer-associated genes among Sub-GOFA, Fisher's exact test for GO, and Fisher's exact test for gene symbols.

\section{Results \& Discussion:}

Table S1 shows the number and proportion of males and females in the case and control groups, as well as the mean and standard deviation of age (see supplementary material). The analysis dataset does not show a significant difference in gender and age between the case and control groups under clonal mosaicism. In bladder cancer's case groups, $21.3 \%$ of the patients were males, $78.7 \%$ were females, and the age was $69.3 \pm 6.71$. Prostate cancer is a male cancer, and the age of the case group was $71.01 \pm 6.72$.In lung cancer's case groups, $52.1 \%$ were males, $47.8 \%$ were females, and the age was $67.25 \pm 9.5$.The control groups in the three cancer types of analysis dataset did not differ significantly from the case groups in terms of gender ratio and age. In addition, Figure 2also shows a comparison of the frequency of clonal mosaicism events at $1000 \mathrm{Mb}$ for each chromosome in the case and control groups in those ageand gender-adjusted analysis dataset.

Sub-GOFA has superior performance in detecting cancer-associated genes compared to conventional statistical methods. We evaluated the performance of Sub-GOFA by comparing the detection ratio of lung cancer-associated genes obtained from DisGeNET with different FDR thresholds of Sub-GOFA and conventional statistical methods, Fisher's exact test for gene symbol and for GO, in the lung analysis dataset(Figure 1B).The ratio of lung cancer-associated genes detected by Sub-GOFA increased when strict FDR threshold values wereapplied, from $6.74 \%$ at FDR value of 0.5 to $12.9 \%$ at FDR value of 0.1 . On the other hand, Fisher's exact test for GO showed a slight increase from $6.55 \%$ at an FDR value of 0.5 to $6.86 \%$ at FDR value of 0.1 .Fisher's exact test for gene symbols was $2.75 \%$ at FDR value of 0.5 , and all gene symbols were rejected at FDR value of 0.4 and later, resulting in "not applicable" (NA). Sub-GOFA is a genetic functional analysis that also considers low-frequency GO terms.Figure1C shows a plot of the frequency and density of GO terms calculated by Sub-GOFA and Fisher's exact test for GO for lung cancer at FDR value of 0.3. The Sub-GOFA plot shows peaks between 0 and 20. On the other hand, the plot of Fisher's exact test for GO peaks between 20 and 40. Sub-GOFA handled more lowfrequency GO terms and detected a higher ratio of lung cancerassociated genes compared to the conventional method.

For all three types of cancers (bladder, prostate and lung cancer), Sub-GOFA showed higher detection performance of cancerassociated genes than the conventional methods even when p-value was set as the threshold. Since the FDR of Sub-GOFA did not show a significant difference for bladder cancer and prostate cancer, we validated the performance of Sub-GOFA and conventional 
statistical methods by comparing the detection ratio of cancerassociated genes at different $\mathrm{p}$-value thresholds. For all three cancer types, Sub-GOFA showed the highest results compared to the conventional method at all setting threshold of p-value (Figure 3). Especially in bladder cancer and lung cancer, as the p-value was tightened, the detection ratio of cancer-associated genes increased. Sub-GOFA has the functionality to extract the genetic functional differences between lung cancer patients and non-patients under clonal mosaicism. Table 1 shows the genetic functions with the 10 FDR values sorted in ascending order that obtained statistical differences between lung cancer patients and non-patients with the Sub-GOFA analysis. 'Protein localization to microtubule' (GO: 0035372, FDR $=0.0373$ ) and 'Negative regulation of histone methylation' (GO: 0031061, FDR = 0.0398) were extracted from SubGOFA.GO:0035372contains MID1 ( $n=16$, p-value=0.0209) genes, which have been reported as lung cancer-associated genes[18]. In a recent study, using blood-derived DNA methylation and gene expression profiles from a prospective lung cancer case-control study in women, $25 \mathrm{CpG}$ lung cancer markers were identified prior to diagnosis [19]. Those existing research support the certainty of the genetic functional analysis by Sub-GOFA.

\section{Conclusion:}

We describe a tool named Sub-GOFA for Sub-Gene Ontology function analysis in clonal mosaicism using semantic similarity. Sub-GOFA measures the semantic (logical) similarity among patients using the sub-GO network structures of various sizes segmented from the gene ontology (GO) for clustering analysis. The sub-GO's root-terms with significant differences are extracted as disease-associated genetic functions. Sub-GOFA selected a high ratio of cancer-associated genes under validation with acceptable threshold.

\section{Acknowledgements:}

We gratefully acknowledge the work of past and present members of our laboratory.

\section{References:}

[1] Schick UM et al. PLoS ONE. 2013 8:e59823 [PMID: 23533652]

[2] Laurie CC et al. Nature Genetics. 2012 44:642 [PMID: 22561516]

[3] Jacobs KB et al. Nature Genetics. 2012 44:651 [PMID: 22561519]

[4] Machiela MJ et al. American Journal of Human Genetics. 2015 96:487 [PMID: 25748358]

[5] MacHiela MJ et al. Nature Communications. 2016 7:11843 [PMID: 27291797]

[6] Schick UM et al. PLoS ONE. 2013 8:e59823 [PMID: 23533652]

[7] Reina-Castillón J et al. Blood Adv. 2017 1:237 [PMID: 29296947]

[8] Guo Y et al. J Med Genet. 2016 53:643 [PMID: 27287394]

[9] Lareau CA et al. Blood Adv. 20193:4161 [PMID: 31841597]

[10] Zhao $Y$ et al. Nat Commun. 202112:4178 [PMID: 34234147]

[11] Bonnefond A et al. Nature Genetics. 2013 45:1040 [PMID: 23852171]

[12] Harris MA et al. Nucleic Acids Research. 2004 32:D258 [PMID:14681407]

[13] Mazandu GK et al. Briefings in bioinformatics. 2017 18:886 [PMID: 27473066]

[14] Lin D, Proc. of the Intl. Conf. on Machine Learning, 1998

[15] Greene D et al. Bioinformatics. 2017 33:1104 [PMID: 28062448]

[16] Durinck S et al. Nature Protocols. 2009 4:1184 [PMID: 19617889]

[17] Piñero J et al. Nucleic Acids Research. 2017 45:D833 [PMID: 27924018]

[18] Zhang L et al. Journal of cancer research and clinical oncology. 2018 144:855 [PMID: 29450633]

[19] Sandanger TM et al. Scientific Reports. 2018 8:16714 [PMID: 30425263] 

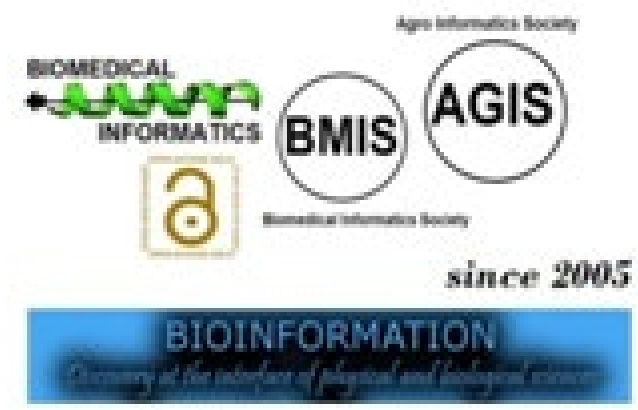

indexed in

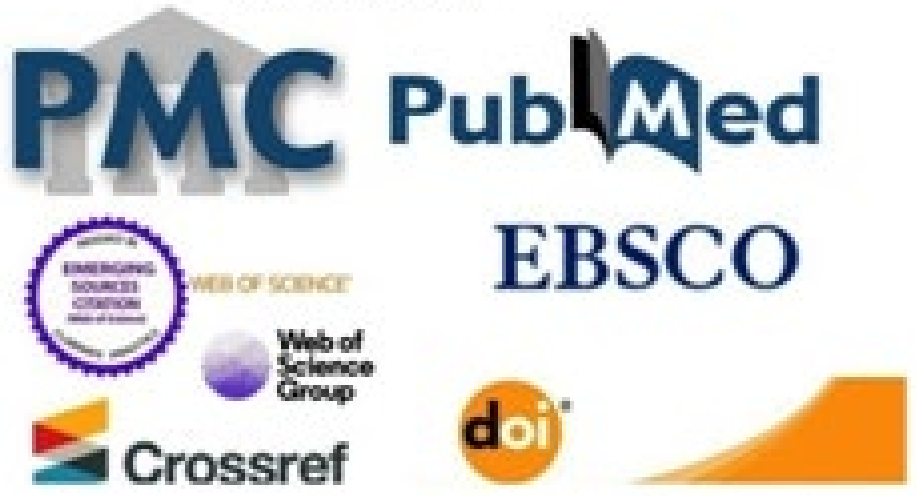

ResearchGate $\mathrm{R}^{\mathrm{G}}$

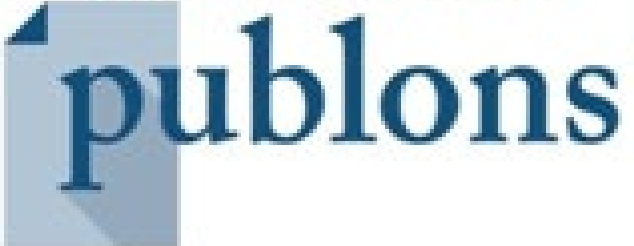

